\title{
Moving Mirrors, Page Curves, and Bulk Entropies in $\mathbf{A d S}_{\mathbf{2}}$
}

\author{
Ignacio A. Reyes $\oplus^{*}$ \\ Max-Planck-Institut für Gravitationsphysik, Am Mühlenberg 1, 14476 Potsdam, Germany
}

(Received 10 March 2021; accepted 23 June 2021; published 29 July 2021)

\begin{abstract}
Understanding the entanglement of radiation in quantum field theory has been a long-standing challenge, with implications ranging from black hole thermodynamics to quantum information. We demonstrate how the case of the free fermion in $1+1$ dimensions reveals the details of the density matrix of the radiation produced by a moving mirror. Using the resolvent method rather than standard conformal field theory techniques we derive the Rényi entropies, modular Hamiltonian, and flow of the radiation and determine when mirrors generate unitary transformations.
\end{abstract}

DOI: 10.1103/PhysRevLett.127.051602

Introduction.-It is well known that the physics of moving mirrors in quantum field theory is intimately connected - and in some cases equivalent - to the thermodynamics of black holes. This relation has proven very fruitful, as the former does not require involved geometric considerations but rather only some fundamental notions about quantum fields.

Two main strategies have been traditionally used in this subject. The first one consists of studying the global properties of the asymptotic state and resembles more closely Hawking's original calculation [1-8]. More recently, techniques coming from gauge-gravity duality, specifically the Ryu-Takayanagi formula and its generalizations [9-11], allow translation of the problem into gravitational physics in a higher dimensional space [12-14]. This second approach relies strongly on methods of conformal field theory. Yet despite significant progress, some important questions remain open.

Often the above approaches are restricted to studies of entanglement entropy. However, a quantum state is not determined only by its entanglement entropy. What one would like to figure out is the structure of the density matrix itself as the system evolves. This means that we seek to understand the correlations between arbitrary subsystems of the radiation, a property that is neither global nor fixed by conformal symmetry. In this work we do precisely this for a very simple system: the chiral fermion in $1+1$ dimensions with a reflecting boundary. The advantage is that here we have the luxury of using the method of the resolvent [15].

Published by the American Physical Society under the terms of the Creative Commons Attribution 4.0 International license. Further distribution of this work must maintain attribution to the author(s) and the published article's title, journal citation, and DOI. Funded by SCOAP.
Finding the Rényi entropies is particularly important in connection with the information paradox. Indeed, unitarity requires that not only the von Neumann entropy but all Rényi entropies follow a Page curve. We will see under which conditions this is true for moving mirrors and quantify the correlations between the early and late radiations. A key ingredient will be the entanglement between the two chiralities created by the mirror.

We begin by specifying the physical system and stating the questions we wish to address.

Fermions and mirrors.-We consider the standard massless Dirac action over a patch $\mathcal{M}$ of $1+1$-dimensional Minkowski spacetime. As usual, using light cone coordinates $x^{ \pm}=t \pm x$ this reads

$$
I=\frac{i}{2} \int_{\mathcal{M}} d x d t\left(\psi_{-}^{\dagger} \partial_{+} \psi_{-}+\psi_{+}^{\dagger} \partial_{-} \psi_{+}\right)
$$

We are interested in the case when $\mathcal{M}$ has a boundary $\partial \mathcal{M}$ along a worldline specified by a differentiable monotonically increasing function

$$
x^{+}=g\left(x^{-}\right)
$$

and we will demand $g^{\prime}>0$ so that its trajectory is causal. For definiteness, we choose the physical region to be that on the right of the boundary, so that incoming (outgoing) modes correspond to left (right) movers $\psi_{+}\left(\psi_{-}\right)$.

For any equations of motion to follow from an action principle, we must require that the action has an extremum. On variation, the action gives $\delta I=\int_{\mathcal{M}}$ e.o.m. $+B$. The first term involves the equations of motion so it vanishes whenever $\partial_{ \pm} \psi_{\mp}=0$, while the second term is a total derivative,

$$
B=\frac{i}{2} \int_{\partial \mathcal{M}} d x^{-}\left(\psi_{-}^{\dagger} \delta \psi_{-}-g^{\prime}\left(x^{-}\right) \psi_{+}^{\dagger} \delta \psi_{+}\right)+\text {H.c. }
$$


and must be required to vanish. A natural condition that achieves $B=0$ is that $\partial \mathcal{M}$ acts as a "mirror" by imposing reflecting boundary conditions,

$$
\psi_{-}\left(x^{-}\right)=\epsilon \sqrt{g^{\prime}\left(x^{-}\right)} \psi_{+}\left(g\left(x^{-}\right)\right) .
$$

Incoming modes reaching the boundary (mirror) are reflected as outgoing modes, the choice $\epsilon= \pm 1$ corresponding to whether the wave flips orientation on reflection.

We will not consider standard boundary conformal field theory (BCFT) [16] because arbitrary mirror trajectories in general break all conformal symmetries. In other words, the boundary conditions (4) are not "conformal boundary conditions": although the stress tensor remains traceless due to the equations of motion, its parallel-perpendicular component-which measures the energy flowing away at the boundary-does not vanish but is governed by the anomaly. If the incoming state is the vacuum, this gives

$$
\left.\left\langle: T_{\| \perp}:\right\rangle\right|_{\text {mirror }}=\frac{(S g)(z)}{12 \pi g^{\prime}(z)},
$$

where $S g$ stands for the Schwarzian derivative. Thus energy will be injected or extracted to or from the system by the moving mirror, similar to what the gravitational field does to the quantum fields outside the horizon. As initial data, we must provide the quantum state on some Cauchy surface. For simplicity we focus on mirrors that were asymptotically static in the past, so that the incoming state is well defined along past null infinity $\mathcal{I}_{R}^{-}$; see Fig. 3 .

In a free theory, the two-point function plays a major role. Throughout the text we denote by

$$
G(x, y) \equiv\left\langle\psi_{+}(x) \psi_{+}^{\dagger}(y)\right\rangle
$$

the incoming-incoming (left-left) correlation function, i.e., the initial data on $\mathcal{I}_{R}^{-}$. Here $x, y$ are spatial coordinates along a Cauchy slice as described below. Although our analysis is valid for a larger class of Gaussian states, we focus on incoming equilibrium states. This simplifies the discussion and emphasizes the role of the mirror rather than the initial data. An incoming state prepared on $\mathcal{I}_{R}^{-}$at inverse temperature $\beta$ is given by

$$
G(x, y)=\frac{1}{2 i \beta \sinh [\pi(x-y) / \beta]} .
$$

Because both chiralities are involved, we must consider the correlation matrix for the Dirac spinor $\Psi=\left(\psi_{+}, \psi_{-}\right)$,

$$
\mathbf{G}\left(x_{1}, x_{2}\right)=\left\langle\Psi\left(x_{1}\right) \Psi^{\dagger}\left(x_{2}\right)\right\rangle=\left(\begin{array}{ll}
G_{++} & G_{+-} \\
G_{-+} & G_{--}
\end{array}\right),
$$

where $G_{i j} \equiv\left\langle\psi_{i} \psi_{j}^{\dagger}\right\rangle$ with $i, j= \pm$. Two-dimensional spinor matrices are denoted in boldface. While $G_{++}$is initial data, the boundary conditions (4) determine the remaining entries of the correlator in terms of $G$ :

$G_{i j}=\epsilon^{[(i-j) / 2]} \sqrt{(-1)^{[(i-j) / 2]} q_{i}^{\prime}(x) q_{j}^{\prime}(y)} G\left[q_{i}(x), q_{j}(y)\right]$,

where we have defined $q_{+}(x) \equiv x^{+}, q_{-}(x) \equiv g\left(x^{-}\right)$.

We now choose an incoming state specified by (7) and a mirror trajectory specified by $g(\cdot)$. Together these determine a global state $\rho_{\Sigma}$ on each Cauchy slice $\Sigma$; see Fig. 1. We now choose a subregion $V \subset \Sigma$ consisting of $N$ disjoint intervals $V=\mathrm{U}_{\ell}\left(a_{\ell}, b_{\ell}\right)$ with $\ell=1, \ldots, N$ and wish to compute the reduced density matrix

$$
\rho_{V}=\operatorname{Tr}_{V^{c}}\left(\rho_{\Sigma}\right)
$$

obtained by tracing out the complement of $V$ along $\Sigma$. For simplicity we restrict to Cauchy slices of constant time, the generalization being straightforward [17].

Here is where having a free theory comes in handy [18]. For free fermions, it is sufficient that $\rho_{V}$ reproduces the correlator $\mathbf{G}(x, y)=\operatorname{tr}_{V}\left[\rho_{V} \Psi(x) \Psi^{\dagger}(y)\right]$ for $x, y \in V$. Now any state can be written as $\rho_{V}=e^{-K_{V}}$, where $K$ is called the modular Hamiltonian. For Gaussian states this takes a quadratic form,

$$
K=\int_{V^{2}} d x d y \Psi^{\dagger}(x) \mathbf{k}(x, y) \Psi(y)
$$

with kernel [18]

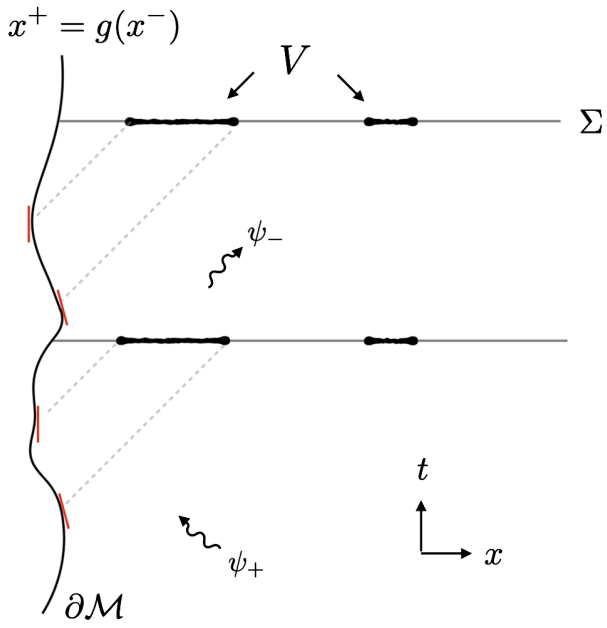

FIG. 1. Evolution of an entangling region $V$ for a given mirror trajectory. The Rényi entropies of $V$ depend on the mirror only through the position and velocity at the null projections of the region's boundary, as exemplified in (18). Thus if these return to their original values, the mirror produces unitary transformations. 


$$
\mathbf{k}=-\log \left(\mathbf{G}^{-1}-\mathbf{1}\right)
$$

Here and below $\mathbf{G}(x, y)$ is taken as a linear operator acting on smooth functions supported on $V$ via convolution.

This translates the problem of finding the reduced density matrix into that of computing functions of $\mathbf{G}$ on $V$, but with both chiralities simultaneously. This sets the stage for the application of the resolvent method, a technical tool that is reviewed in the Supplemental Material [19]. The basic idea is that to compute functions of an operator one can use Cauchy's integral formula,

$$
f(\mathbf{G})=\frac{1}{2 \pi i} \oint_{\gamma} d \lambda \frac{f(\lambda)}{\lambda-\mathbf{G}}
$$

where $\gamma$ encircles the spectrum of $\mathbf{G}$. The operator $(\lambda-\mathbf{G})^{-1}$ is called the resolvent of $\mathbf{G}$.

The simplest application of this method is to compute the entropies, to which we now turn.

Entropies.-The entanglement Rényi entropies are defined as

$$
S^{(n)}=\frac{1}{1-n} \log \frac{\operatorname{Tr}\left(\rho^{n}\right)}{(\operatorname{Tr} \rho)^{n}} .
$$

For the free fermion, it is easy to show that

$$
\log \operatorname{Tr}\left(\rho^{n}\right)=\operatorname{Tr} \log \left[\mathbf{G}^{n}+(1-\mathbf{G})^{n}\right] .
$$

In the Supplemental Material [19] we show how to use the resolvent to compute these expressions. After the dust settles, the final result for the Rényi entropies is

$$
S^{(n)}=\left.\frac{n+1}{24 n} \log \frac{\Omega\left(x^{+}\right)}{\Omega\left[g\left(x^{-}\right)\right]}\right|_{\partial V_{\delta}},
$$

where

$$
\Omega(x)=-\prod_{\ell=1}^{N} \frac{G\left(x, b_{\ell}^{+}\right)}{G\left(x, a_{\ell}^{+}\right)} \frac{G\left[x, g\left(a_{\ell}^{-}\right)\right]}{G\left[x, g\left(b_{\ell}^{-}\right)\right]}
$$

and we have introduced the region $V_{\delta}=\mathrm{U}_{\ell}\left(a_{\ell}+\delta, b_{\ell}-\right.$ $\delta$ ) with a very small $\delta>0$ to regularize the UV divergences. Throughout the Letter all $n$ dependence of Rényi entropies appears as an overall factor.

Here it is illustrative to look at a specific example. Consider the vacuum as an incoming state and a single interval $(a, b)$ at time $t$ but leaving the mirror trajectory arbitrary. The entropies (16) yield
$S^{(n)}=\frac{n+1}{12 n} \log \left(\frac{b-a}{\delta^{2}} \frac{b^{+}-g\left(b^{-}\right)}{\frac{b^{+}-g\left(a^{-}\right)}{a^{+}-g\left(b^{-}\right)}} \frac{g\left(a^{-}\right)-g\left(b^{-}\right)}{\sqrt{g^{\prime}\left(b^{-}\right) g^{\prime}\left(a^{-}\right)}}\right)$.

This result is remarkably simple. It depends on the mirror position $g$ and velocity $g^{\prime}$ only at $a^{-}, b^{-}$, i.e., where the null projections of $\partial V$ intersect the mirror trajectory; see Fig. 1.

This gives rise to a unitarity criterion in the following way. Consider a fixed interval $(a, b)$ together with a mirror trajectory $g(t)$. If the position and velocity of the mirror at the null projections of $\partial V$ are identical at $t_{1}$ and $t_{2}$, then all entropies (18) at $t_{1}$ are equal to those at $t_{2}$, and the mirror generates states related by a unitary transformation

$$
U \rho_{V}\left[g\left(t_{1}\right)\right] U^{\dagger}=\rho_{V}\left[g\left(t_{2}\right)\right]
$$

The behavior of the mirror anywhere else is irrelevant. This case is depicted in Fig. 1. Here it is crucial to observe that, even if all the Rényi entropies coincide, the states $\rho_{V}\left[g\left(t_{1,2}\right)\right]$ can be very different. Indeed, Gaussian states are determined by their correlation functions, and two different mirrors with identical $g\left(a^{-}\right), g\left(b^{-}\right), g^{\prime}\left(a^{-}\right), g^{\prime}\left(b^{-}\right)$ produce the same entropies but different correlators.

A useful tool to quantify unitarity questions is the mutual information, as we show next.

Mutual information: Mutual information (MI) is defined as $I\left(V_{1} \mid V_{2}\right)=S^{(1)}\left(V_{1}\right)+S^{(1)}\left(V_{2}\right)-S^{(1)}\left(V_{1} \cup V_{2}\right)$. It measures the inherent correlations between $V_{1}$ and $V_{2}$ and is a better quantifier of correlations than entropy because it is UV finite. From (16) we find that the mutual information in the presence of a moving mirror decomposes as

$$
I=I_{\text {plane }}+\frac{1}{6} \log \omega
$$

The first term is the usual mutual information of two independent chiralities on the plane (no boundary), while the second term is the new contribution due to the mirror. To illustrate them, let us consider the simple case of the vacuum as incoming state. Then, we have as usual $I_{\text {plane }}=\frac{1}{6} \log \left\{\left[\left(a_{2}-a_{1}\right)\left(b_{2}-b_{1}\right)\right] /\left[\left(b_{2}-a_{1}\right)\left(a_{2}-b_{1}\right)\right]\right\}$. The novel term due to the mirror is given by

$$
\omega=\frac{\frac{\left(b_{2}^{+}-g\left(a_{1}^{-}\right)\right)\left(b_{1}^{+}-g\left(a_{2}^{-}\right)\right)\left(g\left(b_{2}^{-}\right)-g\left(b_{1}^{-}\right)\right)}{\left(b_{2}^{+}-g\left(b_{1}^{-}\right)\right)\left(b_{1}^{+}-g\left(b_{2}^{-}\right)\right)\left(g\left(b_{2}^{-}\right)-g\left(a_{1}^{-}\right)\right)}}{\frac{\left(a_{2}^{+}-g\left(a_{1}^{-}\right)\right)\left(a_{1}^{+}-g\left(a_{2}^{-}\right)\right)\left(g\left(a_{2}^{-}\right)-g\left(b_{1}^{-}\right)\right)}{\left(a_{2}^{+}-g\left(b_{1}^{-}\right)\right)\left(a_{1}^{+}-g\left(b_{2}^{-}\right)\right)\left(g\left(a_{1}^{-}\right)-g\left(a_{2}^{-}\right)\right)}}
$$

It is noteworthy that although the individual entropies (18) depend on the mirror velocity $g^{\prime}$, mutual information is independent of it. The velocity dependence arises via the UV divergences, which mutual information is devoid of by construction. 
For example, for the static mirror, (21) is given by

$$
\omega_{\mathrm{RHP}}=\frac{\sinh \frac{\pi\left(a_{1}+b_{2}\right)}{\beta} \sinh \frac{\pi\left(a_{2}+b_{1}\right)}{\beta}}{\sinh \frac{\pi\left(a_{1}+a_{2}\right)}{\beta} \sinh \frac{\pi\left(b_{1}+b_{2}\right)}{\beta}} .
$$

In the examples below, mutual information will prove useful to quantify the violation of unitarity produced by different mirrors. Having understood the entropies, we now move on to another important aspect dealing with the action of the density matrix itself.

Modular Hamiltonian and flow.-The modular Hamiltonian (11) defines the modular flow, an automorphism of the algebra of observables supported on the causal region associated to $V$. For any operator $\mathcal{O}$, it is defined by evolving with the modular Hamiltonian

$$
\sigma_{\tau}(\mathcal{O}) \equiv e^{-i \tau K} \mathcal{O} e^{i \tau K}
$$

with $\tau$ the "modular time." The simplest flow to study is that of the fundamental field itself. Using the tools in [20], we find that

$$
\sigma_{\tau}\left(\psi_{i}(x)\right)=\int_{V} d y \Sigma_{i j}(x, y) \psi_{j}(y)
$$

with the modular kernel $\mathbf{\Sigma}=\left(\mathbf{G}^{-1}-1\right)^{-i \tau}$. And once more the resolvent allows to compute this; the result is

$\Sigma_{i j}=2 \pi i \sinh (\pi \tau) \delta\left(Z\left[q_{i}(x)\right]-Z\left[q_{j}(x)\right]-\tau\right) G_{i j}(x, y)$.

To understand the locality properties of the flow, we must examine the number and nature of the solutions to

$$
Z\left[q_{i}(x)\right]-Z\left[q_{j}(y)\right]-\tau=0 .
$$

For our purposes only one property of the function $Z(\cdot)$ is relevant: $Z\left(q_{i}\right)$ for $i= \pm$ increases/decreases monotonically from $\pm \infty$ to $\mp \infty$ in each interval of $V$. Therefore, there exists a unique solution to (26) in each interval as follows. For equal chiralities, $i=j$, we call the solutions $y_{\ell}(x)$, where $\ell$ labels the interval. These are similar to those already encountered in [15,20-22], and include the local solution $y=x$. For opposite chiralities $i=-j$, we have a novel set of solutions that we call $y_{-\ell}$ to indicate that they are associated with a change in chirality.

Introducing the kernel (25) into (24) yields the explicit form of the modular flow:

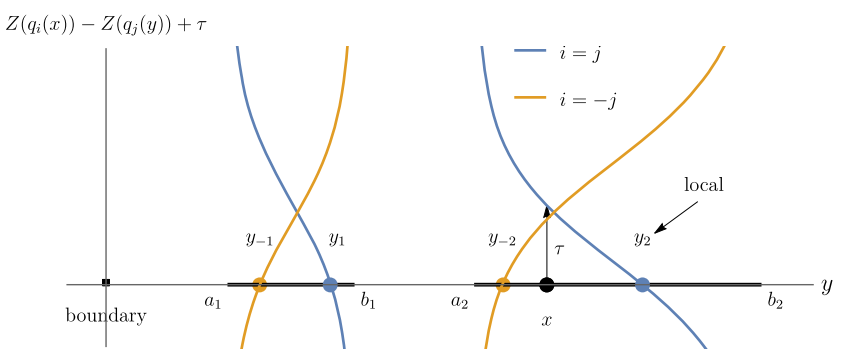

FIG. 2. Illustration of the modular evolution governed by (24), for two disjoint intervals and a static mirror and incoming vacuum. We plot (26) for equal and opposite chiralities (this is not a spacetime diagram). The modular flow of $\psi_{i}(x)$ yields, at each interval, contributions of both chiralities $\psi_{ \pm i}$ located at the points $y_{ \pm \ell}(x)$ that are solutions to (26) (colored circles). Evolution in modular time $\tau$ shifts the curves vertically, and the roots evolve accordingly. The root at $y_{2}$ is the local (geometric) solution, with $y_{2} \rightarrow x$ as $\tau \rightarrow 0$. Here $a_{1}=1, b_{1}=2$, $a_{2}=3, b_{2}=5.3$.

$$
\begin{aligned}
\sigma_{\tau}\left[\psi_{i}(x)\right]= & 2 \pi \sinh (\pi \tau) \sum_{\ell}\left(\frac{G_{i i}\left[x, y_{\ell}(x)\right] \psi_{i}\left[y_{\ell}(x)\right]}{\left|\partial_{y} Z(y) \partial_{y} q_{i}(y)\right|_{y=y_{\ell}(x)}}\right. \\
& \left.+\frac{G_{i,-i}\left[x, y_{-\ell}(x)\right] \psi_{-i}\left[y_{-\ell}(x)\right]}{\left|\partial_{y} Z(y) \partial_{y} q_{-i}(y)\right|_{y=y_{-\ell}(x)}}\right) .
\end{aligned}
$$

Modular flow takes the field of chirality $i$ at point $x$ and evolves it to two points on each interval: a contribution of the same chirality located at $y_{\ell}(x)$ and another with opposite chirality located at $y_{-\ell}(x)$; see Fig. 2.

After these formal developments, we turn our attention to some specific examples of mirror trajectories that are of particular physical interest.

Examples of mirrors. - A static mirror: As our simplest example, consider a single interval on the right half plane (RHP), i.e., a static boundary at $x=0$. This does give rise to a conformal boundary condition where (5) vanishes. For the vacuum as incoming state, the Rényi entropies (16) give

$$
S_{\mathrm{RHP}}^{(n)}=\frac{n+1}{12 n}\left[\log \left(\frac{b-a}{\delta}\right)^{2}+\log \frac{4 r}{(r+1)^{2}}\right],
$$

where $r=b / a$. These agree with those reported in [23] recently. The first term is simply twice the universal entropy of a single chirality, while the second one is due to the mirror. If the incoming state is thermal, the entropies are instead

$S_{\mathrm{RHP}}^{(n)}=\frac{n+1}{12 n}\left[\log \left(\frac{\beta}{\pi \delta} \sinh \frac{\pi(b-a)}{\beta}\right)^{2}+\log \frac{4 \tilde{r}}{(\tilde{r}+1)^{2}}\right]$,

where $\tilde{r}=\tanh [(2 \pi a) / \beta] / \tanh [(2 \pi b) / \beta]$. Notice that the second term in both (28) and (29) is always negative so the mirror lowers the entropy of the system. 
Moving now to the modular flow, the case of two intervals $V=\left(a_{1}, b_{1}\right) \cup\left(a_{2}, b_{2}\right)$ is depicted in Fig. 2. As we saw above, Eq. (26) determines which points are coupled along the flow. In this case (26) is a quartic equation yielding four real solutions $y_{ \pm 1,2}$. For more intervals, the situation is completely analogous, involving two points per interval.

From vacuum to thermal and back: Next, we focus on an accelerating trajectory that shares the characteristic feature of Hawking radiation, namely that the outgoing state measured at $\mathcal{I}^{+}$is thermal. In our moving mirror setup, there is a unique mirror profile that takes an incoming vacuum and renders the outgoing modes in a thermal state. This is

$$
g_{\mathrm{I}}\left(x^{-}\right)=\frac{\beta}{\pi} \tanh \left(\frac{\pi}{\beta} x^{-}\right) \quad \text { vacuum } \rightarrow \text { thermal. }
$$

Because this trajectory becomes null also in the remote past producing singularities, its early stage must be replaced by a timelike one as we have mentioned above; see Fig. 3. As is well known [2], this class of trajectories is closely related to the exterior region of a black hole formed by collapse, with the associated problem of information loss.

Interestingly, the converse effect is also possible: given an incoming thermal state, any trajectory that asymptotes to the inverse function, namely $\tilde{g}_{\mathrm{I}}\left(x^{-}\right)=$ $(\beta / \pi) \operatorname{arctanh}\left[(\pi / \beta) x^{-}\right]$, takes a thermal state and reflects it as the vacuum.

Mirror with uniform acceleration: Our final example is a mirror moving with constant proper acceleration. The mirror stands static at $x=-R$ until $t=0$ when it begins to accelerate at a constant rate away from the physical region, following the Rindler trajectory $t^{2}-x^{2}=-R^{2}$ corresponding to

$$
g_{\mathrm{III}}\left(x^{-}\right)=-R^{2} / x^{-} .
$$

Although qualitatively this profile is similar to $g_{I}$, it exhibits an important difference. The Rényi entropies for $t \gg b-R$ read

$S^{(n)}=\frac{n+1}{12 n}\left[\log \left(\frac{b-a}{\delta}\right)^{2}+\frac{2 R^{2}-(a+b)^{2} R^{2}+2(a b)^{2}}{t^{4}}\right]$.

This result displays an interesting feature. Notice the first term is identical to the vacuum Rényi entropy of two independent chiralities, as if the mirror was not present. In the asymptotic future, the second term vanishes. Thus the original entanglement between the two chiralities, created by the static mirror, is "erased" exactly by the accelerating mirror, leaving two unentangled chiralities. This is also seen directly by looking back at the correlation matrix itself, for in the limit $t \rightarrow \infty$

$$
G_{ \pm \mp} \rightarrow 0 \text { for } g_{\mathrm{III}}
$$

so that the correlations between left and right movers vanish. Because opposite chiralities are not entangled with each other any more on $V$, they become more entangled with the complement, which has the effect of increasing the entropy. This is a hallmark of nonunitarity: the entropy in the distant future is larger than its counterpart in the remote past.

Page curves from mutual information:()How can we capture the correlations between the early and late radiation? Consider again two fixed disjoint regions $V=V_{1} U$ $V_{2}$ as depicted in Fig. 3. We will compare two mirror trajectories that remain static until $t=0$, when they begin to move. The first is $g_{I}$, already introduced, which scatters the vacuum into a thermal outgoing state. The second, $g_{\mathrm{II}}$, follows $g_{I}$ for some time but then deviates from it in order to smoothly return to the static path. The precise functional form of $g_{\mathrm{II}}$ is irrelevant.

In principle one could simply track the entropies of these regions of space as they evolve in time as done above. However, this approach is not completely satisfactory. First, Rényi entropies are not well defined in the UV. Moreover, if we wish to keep track of all the radiation that has escaped to infinity, we must consider an unbounded spatial subregion, which introduces yet another divergence.

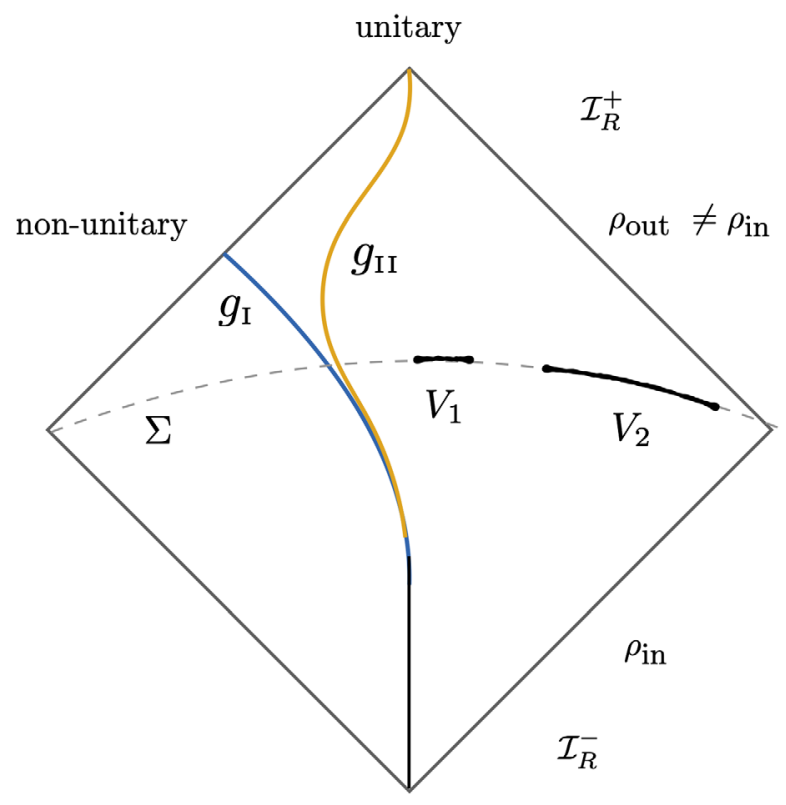

FIG. 3. Two different mirror trajectories with entangling region $V=V_{1} \cup V_{2}$. Although the incoming and outgoing states $\rho_{\text {in/out }}$ are very different, unitarity means that their Rényi entropies on arbitrary $V$ match. Illustrated are $g_{I}$ and $g_{\text {II }}$ from the main text: the former starts to accelerate, becoming asymptotically null, describing a nonunitary process. The latter follows the former for some time before returning to its original trajectory and respects unitarity. 
$-g_{\mathrm{I}}$, non-unitary $-g_{\mathrm{II}}$, unitary

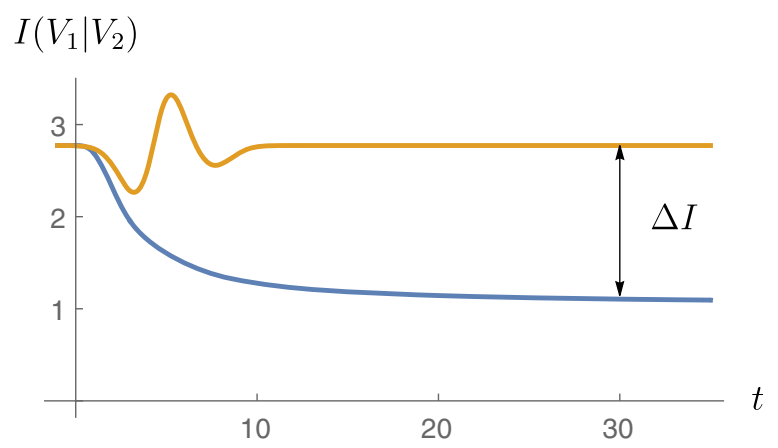

FIG. 4. Evolution of mutual information (20) between the regions $V_{1}$ and $V_{2}$, for the two mirror trajectories depicted in Fig. 3, measuring the amount of correlations between the early and late radiation. MI returns to its original value for trajectory $g_{\mathrm{II}}$, whereas it does not for $g_{\mathrm{I}}$. The loss of correlations $\Delta I$ given in (34) serves to quantify unitarity violation. Here $a_{1}=0, b_{1}=1$, $a_{2}=3, b_{2}=10^{6}$ with $\beta=7$ in (30).

These problems are remedied by considering mutual information instead. MI is always finite: it is by construction free from UV divergences, and in addition we can safely take the limit $b_{2} \rightarrow \infty$ that stretches all the way to spatial infinity. Furthermore, MI has the natural physical interpretation that we seek: it measures the correlations between the early radiation (collected in $V_{2}$ ) and the late radiation (contained in $V_{1}$ ).

In Fig. 4 we plot the evolution of the MI between the early and late radiation as a function of time. Clearly for the trajectory $g_{I}$, there is a loss of correlations between the late and early radiation compared to trajectory $g_{\mathrm{II}}$, which is unitary. The asymptotic difference in MI, $\Delta I=$ $\lim _{t \rightarrow \infty} \lim _{b_{2} \rightarrow \infty}\left[I_{g_{\text {II }}}\left(V_{1} \mid V_{2}\right)-I_{g_{\mathrm{I}}}\left(V_{1} \mid V_{2}\right)\right]$, can be used to quantify the violation of unitarity as a function of the temperature of the outgoing radiation and reads

$\Delta I=\frac{1}{3} \log \frac{\left(a_{2}-a_{1}\right)\left(a_{2}+b_{1}\right)^{2}\left(e^{\left[\left(2 \pi a_{2}\right) / \beta\right]}-e^{\left[\left(2 \pi b_{1}\right) / \beta\right]}\right)}{\left(a_{2}-b_{1}\right)\left(a_{1}+a_{2}\right)^{2}\left(e^{\left[\left(2 \pi a_{2}\right) / \beta\right]}-e^{\left[\left(2 \pi a_{1}\right) / \beta\right]}\right)}$.

It increases monotonically until saturating at high temperature.

Conclusion.-In this Letter we have investigated the entanglement in the radiation produced by a moving mirror using analytic techniques. The main physical effect of introducing a reflecting boundary is that of entangling the two chiralities. This shows up as extra terms in the Rényi entropies and in the modular flow as additional bilocal couplings due to chirality exchange. We found that for a static mirror, the entanglement among the chiralities always decreases the entropies. It would be very interesting to understand this in the context of monogamy of entanglement in quantum field theory [24].

It is a pleasure to thank Ana Alonso-Serrano, Enrico Brehm, Raghu Mahajan, Juan Maldacena, Pedro F. Ramirez, and the GQFI group at AEI for stimulating discussions. In particular I wish to thank Raúl Arias and Rob Myers for insightful comments on an early version of the draft. I would especially like to thank Pascal Fries for joining the initial stages of the project as well as elucidating some subtle aspects. The Gravity, Quantum Fields and Information group at AEI is generously supported by the Alexander von Humboldt Foundation and the Federal Ministry for Education and Research through the Sofja Kovalevskaja Award.

*ignacio.reyes@aei.mpg.de

[1] S. W. Hawking, Black hole explosions, Nature (London) 248, 30 (1974).

[2] P. C. W. Davies and S. A. Fulling, Radiation from a moving mirror in two-dimensional space-time conformal anomaly, Proc. R. Soc. A 348, 393 (1976).

[3] P. C. W. Davies and S. A. Fulling, Radiation from moving mirrors and from black holes, Proc. R. Soc. A 356, 237 (1977).

[4] L. H. Ford and A. Vilenkin, Quantum radiation by moving mirrors, Phys. Rev. D 25, 2569 (1982).

[5] P. Chen and D.-h. Yeom, Entropy evolution of moving mirrors and the information loss problem, Phys. Rev. D 96, 025016 (2017).

[6] M. R. R. Good, E. V. Linder, and F. Wilczek, Moving mirror model for quasithermal radiation fields, Phys. Rev. D 101, 025012 (2020).

[7] M. R. R. Good, K. Yelshibekov, and Y.C. Ong, On horizonless temperature with an accelerating mirror, J. High Energy Phys. 03 (2017) 013.

[8] M. Hotta, M. Shino, and M. Yoshimura, Moving mirror model of Hawking evaporation, Prog. Theor. Phys. 91, 839 (1994).

[9] S. Ryu and T. Takayanagi, Holographic Derivation of Entanglement Entropy from AdS/CFT, Phys. Rev. Lett. 96, 181602 (2006).

[10] V.E. Hubeny, M. Rangamani, and T. Takayanagi, A covariant holographic entanglement entropy proposal, J. High Energy Phys. 07 (2007) 062.

[11] T. Faulkner, A. Lewkowycz, and J. Maldacena, Quantum corrections to holographic entanglement entropy, J. High Energy Phys. 11 (2013) 074.

[12] A. Almheiri, N. Engelhardt, D. Marolf, and H. Maxfield, The entropy of bulk quantum fields and the entanglement wedge of an evaporating black hole, J. High Energy Phys. 12 (2019) 063.

[13] A. Almheiri, R. Mahajan, J. Maldacena, and Y. Zhao, The Page curve of Hawking radiation from semiclassical geometry, J. High Energy Phys. 03 (2020) 149.

[14] I. Akal, Y. Kusuki, N. Shiba, T. Takayanagi, and Z. Wei, Entanglement Entropy in a Holographic Moving Mirror and the Page Curve, Phys. Rev. Lett. 126, 061604 (2021). 
[15] H. Casini and M. Huerta, Reduced density matrix and internal dynamics for multicomponent regions, Classical Quantum Gravity 26, 185005 (2009).

[16] P. Di Francesco, P. Mathieu, and D. Senechal, Conformal Field Theory, Graduate Texts in Contemporary Physics (Springer-Verlag, New York, 1997).

[17] In the continuum limit the existence of the density operator is of course problematic. In this paper we abuse notation and use this notation since it is the more familiar language.

[18] I. Peschel, Calculation of reduced density matrices from correlation functions, J. Phys. A 36, L205 (2003).

[19] See Supplemental Material at http://link.aps.org/ supplemental/10.1103/PhysRevLett.127.051602 for technical aspects that are not essential for the understanding of the main text. It includes the derivation of the resolvent for the moving mirror, and the application to quantum extremal surfaces in $\mathrm{AdS}_{2}$.

[20] J. Erdmenger, P. Fries, I. A. Reyes, and C. P. Simon, Resolving modular flow: A toolkit for free fermions, J. High Energy Phys. 12 (2020) 126.

[21] I. Klich, D. Vaman, and G. Wong, Entanglement Hamiltonians and entropy in $1+1 \mathrm{D}$ chiral fermion systems, Phys. Rev. B 98, 035134 (2018).

[22] D. Blanco and G. Pérez-Nadal, Modular Hamiltonian of a chiral fermion on the torus, Phys. Rev. D 100, 025003 (2019).

[23] M. Mintchev and E. Tonni, Modular Hamiltonians for the massless Dirac field in the presence of a boundary, J. High Energy Phys. 03 (2021) 204.

[24] We thank Pascal Fries for pointing out this interpretation. 